\title{
La mise en tourisme et le développement local par la création d'une atmosphère gastronomique
}

Analyse à partir du cas de Vonnas

\section{Cécile Clergeau et Olivier Etcheverria}

\section{OpenEdition}

\section{Journals}

Édition électronique

URL : http://journals.openedition.org/tourisme/185

DOI : $10.4000 /$ tourisme. 185

ISSN : 2492-7503

\section{Éditeur}

Éditions touristiques européennes

Édition imprimée

Date de publication : 1 juin 2013

Pagination : 52-67

ISSN : 2109-5671

\section{Référence électronique}

Cécile Clergeau et Olivier Etcheverria, « La mise en tourisme et le développement local par la création d'une atmosphère gastronomique », Mondes du Tourisme [En ligne], 7 | 2013, mis en ligne le 30 septembre 2015, consulté le 19 avril 2019. URL : http://journals.openedition.org/tourisme/185 ; DOI 10.4000/tourisme.185

\section{(c) (i) $\odot$}

Mondes du tourisme est mis à disposition selon les termes de la licence Creative Commons Attribution - Pas d'Utilisation Commerciale - Pas de Modification 4.0 International. 


\section{La mise en tourisme et le développement local}

\section{par la création d'une atmosphère gastronomique}

\section{Analyse à partir du cas de Vonnas}

\section{Cécule Clergeau}

[cecile.clergeau@univ-angers.fr]

Professeur des universités en sciences de gestion

Granem • Université d'Angers

\author{
OLIVIER ETCHEVERRIA \\ [olivier.etcheverria@univ-angers.fr] \\ Maître de conférences en géographie \\ UMR ESO • Université d'Angers
}

Résumé. Vonnas montre l'exemple d'une mise en tourisme et d'une dynamique de développement local portées par un cuisinier entrepreneur, Georges Blanc, qui ancre son parcours économique et son orientation culinaire dans une longue tradition familiale. La stratégie de développement socioéconomique et culturelle du cuisinier, fondée sur l'innovation entrepreneuriale et sur la créativité culinaire, a conduit à l'émergence d'un lieu caractérisé par une singulière concentration d'activités orientées vers la gastronomie : le Village Gourmand. Fondée sur l'identité bressanne, cette atmosphère gastronomique favorise la mise en place des conditions nécessaires au développement local. Georges Blanc va engager une théâtralisation de cette atmosphère gastronomique afin de la rendre pleinement accessible et compréhensible par les touristes. Cette théâtralisation autorise la co-production de l'expérience touristique et, finalement, de la destination touristique gourmande.

Abstract. Vonnas is an interesting example of a touristification process help by an entrepreneur: the well-known chef Georges Blanc. Georges Blanc has inherited a long familial tradition of cuisine. He re-invented his mother and grand-mother's famous dishes and proposed a new way of cooking local products. More, he participated in the initiatives to promote local products, such as the Bressan Poultry. Inspired by the great designer R. Loewy, he also wanted to offer tourists the staging of his culinary art: he created a place, inside Vonnas, called the "Village Gourmand" in witch tourists may experience gastronomy. The staging of gastronomic atmosphere transforms Vonnas in a tourist destination. 
I 1 n'est pas aujourd'hui une recherche ni une politique publique qui ne propose d'appuyer le développement local sur la mise en réseau des acteurs, la valorisation de la culture et de l'identité locales et de ce qu'Alfred Marshall ( 1890 ) appelait une "atmosphère industrielle", c'est-à-dire un ensemble de savoirs partagés, de valeurs, de normes, de traditions qui animent un territoire, en particulier le marché du travail local et les relations sociales dans les entreprises, et favorisent créativité et innovation. La mise en tourisme des territoires à partir de leurs activités économiques interroge les chercheurs sur la transformation de ces atmosphères locales en atouts et attraits touristiques, c'est-à-dire sur l'aptitude des acteurs des territoires à partager avec des habitants temporaires ces savoirs, ces normes et ces valeurs, et à inscrire leur culture et leur identité dans un projet touristique. Nous savons finalement peu de choses sur cette transformation, sur son processus même. Or sa caractérisation, son mode d'émergence et ses dynamiques sont indispensables, nous semble-t-il, à la compréhension de la création des ressources d'un territoire. C'est ce que propose d'explorer cette recherche à travers l'analyse du façonnage de ce que nous nommons une "atmosphère gastronomique".

Nous présentons un cas de mise en tourisme portée par un cuisinier entrepreneur, Georges Blanc, qui ancre son parcours économique dans une longue tradition familiale. Celleci a contribué à l'émergence d'un lieu caractérisé par la densité et la diversité des activités économiques orientées vers la cuisine et le goût et qui se singularise par l'accumulation locale de savoirs et de connaissances gastronomiques. Toute la démarche stratégique de G. Blanc a été de rendre ces activités, d'une part, et ces savoirs et ces connaissances, d'autre part, accessibles aux touristes, leur permettant des expériences mémorables. Nous définissons ainsi l'atmosphère gastronomique comme l'accumulation locale de savoirs, de connaissances, de pratiques, de discours et d'imaginaires gastronomiques et liés au goût qui favorise l'apprentissage et l'acquisition de compétences par les protagonistes de la co-construction de l'expérience touristique. Nous montrons que la mise en tourisme de Vonnas a été réalisée par transformation d'une atmosphère économique locale en une atmosphère gastronomique.

Nous avons opté pour une démarche de recherche qualitative par étude de cas. Cette approche se révèle particulièrement adaptée en réponse à des questions de type “pourquoi ?" ou "comment ?" (Eisenhardt, 1989 ; Yin, 2003). L'enquête doit alors se fonder sur de nombreuses modalités de collecte conduisant à une convergence, selon le principe de la triangulation des données (Yin, 2003). Après un travail de recueil de données secondaires, une période d'observation participante s'est déroulée à Vonnas en juillet 2012. Sept entretiens libres d'une durée de deux à trois heures ont été conduits avec Georges Blanc, avec le maire de Vonnas, l'adjointe au maire chargée de la culture, les acteurs locaux du tourisme et la représentante du Comité interprofessionnel de la volaille de Bresse (CIVB). L'analyse s'appuie aussi sur la visite des lieux, ainsi que sur un ensemble de documents transmis par G. Blanc et les acteurs rencontrés. Cette recherche qualitative vise à comprendre un phénomène bien peu théorisé : la transformation d'un lieu gourmand en une destination touristique gourmande. Seule une telle approche pourra nous permettre d'émettre des hypothèses quant aux processus qui façonnent cette transformation du territoire. Notre observation s'inscrit ici dans une démarche "abductive" visant à produire des hypothèses nouvelles (David, Hatchuel et Laufer, 2000).

Notre recherche s'inscrit dans une perspective socio-économique : nous proposons une conception des activités économiques nourrie des concepts d'encastrement (Granovetter, 1985), de réseaux sociaux, de capital social (Bourdieu, 1980) et d'interdépendance des acteurs du territoire. À la suite de Polanyi (1944, [édition en français: 1983]), nous reconnaissons que toute société a une économie et que l'économie ne peut se développer que dans une société (Éloire, 2009). Nous suivons ainsi Granovetter (1985) dans son plaidoyer pour une analyse des phénomènes économiques, qui considère à la fois un certain libre arbitre des individus tout en admettant que ces individus s'inscrivent dans une société et sont encastrés dans des réseaux sociaux. 
Nous considérons ainsi que l'entrepreneur G. Blanc est inséré dans des réseaux sociaux locaux, dispose d'un capital social (qui a ici une dimension familiale de toute première importance), en sorte que finalement son ancrage territorial se confond avec son encastrement relationnel (Nahapiet et Ghoshal, 1998 ; Bolino, Turney et Bloodgood, 2002) : il entreprend là où ses réseaux (professionnels, amicaux, familiaux) sont les plus denses et les plus aptes à favoriser la mobilisation et la création de ressources spécifiques et de savoirfaire (Reix, 2008). Notre analyse de cas nous permet de montrer comment cet ancrage encastrement a permis le façonnage d'une atmosphère gastronomique théâtralisée par G. Blanc qui porte la transformation d'un lieu gourmand en une destination touristique gourmande.

Une première partie s'intéresse à l'ancrage territorial, familial et social du projet entrepreneurial Blanc, une deuxième partie montre le façonnage de l'atmosphère gastronomique et une dernière partie examine la théâtralisation de cette atmosphère gastronomique et la mise en place des conditions nécessaires au développement local.

\section{De L'AUBERGE AU RESTAURANT 3 衣: L'ANCRAGE TERRITORIAL ET L'ENCASTREMENT SOCIAL DE Georges Blanc ET DE SON ENTREPRISE}

L'histoire de l'entreprise Blanc est intimement liée à celle du village de
Vonnas, dans l'Ain, dans lequel vit la famille Blanc depuis 1872. "C'est la plus vieille maison étoilée sans discontinuité depuis 1929 !... Une longue tradition" (interview de G. Blanc). L'histoire familiale montre, comme dans de nombreux cas (Reix, 2008), que l'ancrage de G. Blanc à Vonnas tient tant à l'optimisation des possibilités de mobilisation de ressources qu'à l'attachement affectif et symbolique : "Le premier point, le point essentiel, en ce qui concerne l'entreprise Blanc, c'est que Georges aime son village... et ça, c'est essentiel" (premiers mots de l'interview du maire de Vonnas). Cette histoire montre également l'existence d'un capital social familial (Arrègle, Durand et Very, 2004) fortement mobilisé par G. Blanc et qui fonde pour partie la performance de son entreprise : "Moi, j'ai un enracinement extrêmement profond dans la région... Je l'ai souvent dit à mes confrères: si vous donnez à la région, la région vous le rendra" (interview de G. Blanc).

\section{Un ancrage local ancien, précoce et profond}

Georges Blanc est un cuisinier restaurateur issu d'une très longue lignée de limonadiers charbonniers. Il est ainsi l'héritier de trois générations de mères cuisinières connues et rapidement distinguées par la critique gastronomique.

L'histoire Blanc commence par l'ouverture par une arrière-grandmère d'une très modeste auberge en 1872, sur la place du Champ-deFoire, au cœur du village de Vonnas.
Jean Louis Blanc et son épouse sont qualifiés de "pionniers" sur le site internet de G. Blanc ${ }^{(1)}$ : "[Ils] furent les premiers aubergistes à s'installer près du champ de foire. Principale clientèle de l'époque: les coquetiers qui, arrivant le jeudi au marché en voiture à cheval, se réchauffaient avec une soupe, puis, leurs achats terminés, s'attablaient devant un copieux casse-croûte. La soupe était bonne, le bruit s'en répandit de marché en marché” (Blanc, 2008, pp. 31 38). Le village comptait à l'époque un peu plus de 1600 habitants. L'auberge acquiert rapidement une reconnaissance locale et régionale, reconnaissance qui se nourrit, en les alimentant, des rapports de confiance et de proximité (en termes de cuisine et de préférences gustatives locales) et est à mettre en relation avec la fréquentation des marchés et foires de la région. Elle connaît alors les débuts d'un processus d'appropriation locale et régionale qui permet la constitution d'un fond de clientèle.

Les grands-parents paternels, Élisa et Adolphe Blanc, développent l'auberge dont ils ont hérité. "En 1902, Adolphe Blanc, le fils, qui avait épousé Élisa Gervais, succéda à ses parents. C'est Élisa qui, installée en cuisine, devait rendre célèbre le nom de la Mère Blanc. Elle avait hérité de sa mère Virginie les secrets d'un art culinaire tout de beurre nourri" (Blanc, 2008, p. 38). L'auberge est alors distinguée par les guides gastronomiques, notamment le Guide Michelin qui lui décerne une première étoile en 1929, et la deuxième en 1931. Concomitamment, Élisa Blanc 
est désignée "meilleure cuisinière du monde" par Curnonsky en 1933. Ainsi, au-delà de la renommée régionale, l'auberge acquiert alors une notoriété nationale et même internationale grâce à la reconnaissance du monde professionnel, d'une part, et de la sphère de la critique gastronomique, d'autre part. L'auberge est en effet un lieu de créativité culinaire, où naissent notamment les crêpes vonnassiennes, présentes encore aujourd'hui sur la carte du restaurant. Ce rayonnement est renforcé, dès les années trente, par la fréquentation d'une clientèle touristique, des habitants temporaires que l'on cherche à attirer en proposant notamment un menu dédié. Menu qui est la condition sine qua non pour la participation au "Concours de la bonne cuisine" organisé par le Touring Club de France, auquel le restaurant obtient une deuxième place $^{(2)}$.

Les grands-parents maternels, Paul Tisserand et son épouse, sont boulangers-pâtissiers installés sur la place de l'Église où ils régalent leurs clients de meringues et, surtout, des célèbres galettes bressanes à la crème et au sucre. Les parents, Jean et Paulette Blanc, font perdurer la renommée de l'auberge et maintiennent les conditions nécessaires au processus d'appropriation locale et régionale. En effet, Paulette Blanc, fille de boulanger, réaffirme l'ancrage local de l'auberge en valorisant, par une cuisine paysanne, les produits agricoles alimentaires locaux et en privilégiant les techniques culinaires et les préférences gustatives appréciées à la frontière géographique de la Bresse et de la Dombes. "Une cuisine certes assez classique, une cuisine de terroir, déclinée selon un répertoire propre à notre famille et inspirée de notre région, à base de produits de qualité, extrêmement frais. Des écrevisses à la nage au pouilly-fuissé ; des cuisses de grenouilles (que nous achetions à la douzaine, livrées en sacs, et non au kilo comme il est d'usage aujourd'hui) qui finissaient dans le beurre mousseux et la persillade; des gâteaux de foie blond de poularde, du poulet de Bresse, à la crème bien évidemment, et du gibier de saison que ma mère adorait cuisiner. Ce qui ne l'empêchait pas d'exceller dans la préparation d'un bœuf braisé aux carottes où elle brillait comme personne dans l'assemblage avec des pieds de veau. Un plat dont se régalaient les représentants de commerce qui se retrouvaient en semaine autour de la table d'hôte" (Blanc, 2008, pp. 1617). Le père est quant à lui cafetier fabricant de limonade mais aussi maire de la commune durant deux mandats (deux mandats en tant que maire adjoint et deux mandats comme maire). Son fils, d'ailleurs, reprendra cette tradition d'investissement dans la vie politique et sociale de la région.

\section{Un projet productif fondé sur la créativité culinaire et l'innovation entrepreneuriale}

Georges Blanc naît à Bourg-enBresse le 2 janvier 1943. Il grandit alléché par les odeurs de la cuisine familiale et les goûts sucrés des flans à la vanille et au caramel d'Élisa
Blanc. "Pour mes parents, il était impensable que je ne reprenne pas l'affaire familiale. Ce sont eux qui m'ont poussé à entrer à l'école hôtelière de Thonon-les-Bains ${ }^{(3)}$." Après de très brillantes études à Thononles-Bains dans l'une des écoles hôtelières les plus réputées de France, d'où il sort major de promotion, il réalise un apprentissage professionnel dans les cuisines de deux palaces : la Réserve de Baulieu, à Beaulieusur-Mer et le Grand Hôtel de Divonne-les-Bains. Il y acquiert les bases techniques de la cuisine royale et princière et le goût des produits alimentaires nobles. En 1964 $\mathrm{G}$. Blanc rejoint sa mère en cuisine dans l'auberge familiale, qu'il reprend à son compte en 1968. Il va alors bouleverser à la fois l'orientation culinaire et l'organisation interne de l'auberge familiale : "Ça a été une grande transformation" (interview G. Blanc).

Il développe en effet très rapidement une curiosité, un intérêt et une sensibilité pour la "nouvelle cuisine". G. Blanc va ainsi se spécialiser dans un “art” des sauces ${ }^{(4)}$ modernisé : il va conduire un remarquable travail d'allègement et de raffinement des préparations saucières. Il renouvelle notamment le répertoire des sauces accompagnant les volailles de Bresse et les cuisses de grenouilles qui ne disparaissent bien sûr pas de la carte du nouveau restaurant. Il construit ainsi son identité culinaire et sa renommée gastronomique sur la préparation de sauces fraîches, légères, pointues et concoctées à la minute. S’il se réfère à deux modèles culinaires 


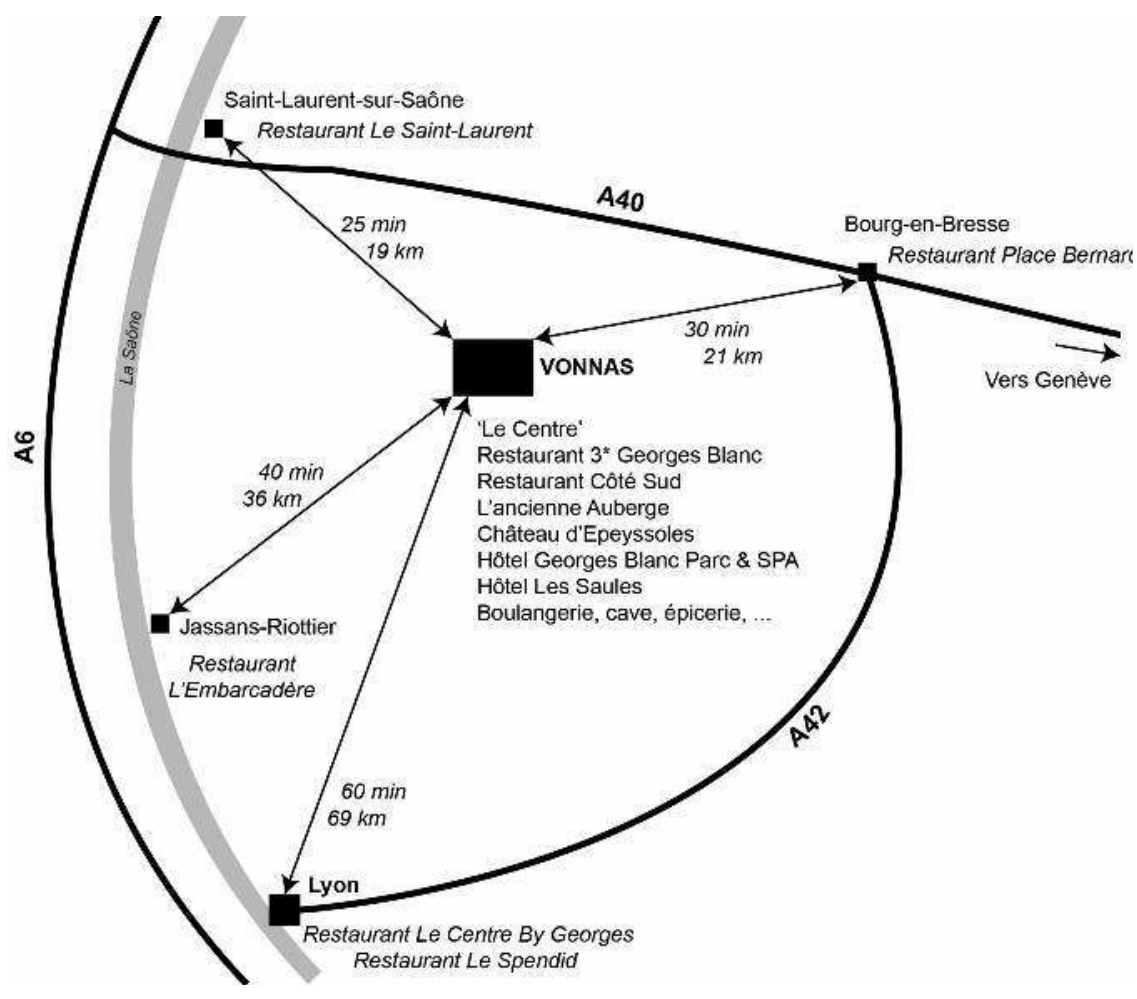

Figure | - L'implantation, selon le modèle centre-périphérie, des établissements du groupe Blanc (20/3)

que sont Alain Chapel, à Mionnay, et Bernard Loiseau, à Saulieu, il continue cependant de puiser dans la tradition familiale et locale son inspiration: "Je me libère un petit peu $d u$ répertoire classique et traditionnel... Je revisite la tradition pour faire une cuisine de création raisonnée mettant toujours en ouvre des produits de qualité avec une priorité aux produits de cette région, notamment l'emblématique poulet de Bresse... Je commence à faire évoluer la cuisine, par exemple la petite crêpe de pommes de terre devient la crêpe au saumon et caviar... le poulet à la crème n'a pas cessé d'évoluer" (inter-
Bernard à Bourg-en-Bresse, Le Splendid à Lyon, L'Embarcadère à Jassans-Riottier, du Château d'Epeyssoles, de deux hôtels, l'hôtel Georges-Blanc et l'hôtel Les Saules, à Vonnas, et de commerces de boulangerie, d'épicerie fine, de cadeauxdécoration-arts de la table à Vonnas ( $c f$. figure 1). Ce groupe dessine un modèle spatial intéressant : celui d'un pôle autour duquel gravitent des périphéries. Il est en effet organisé autour du restaurant 3 次, qui constitue le pôle animé et qui irrigue des flux centripètes et centrifuges. Immédiatement autour de ce pôle central, une première périphérie très proche, structurée autour de la place du Champ-de-Foire où se trouve L'Ancienne Auberge, mais aussi les boutiques, la boulangerie-pâtisserie, les deux hôtels, les lieux de promenade et "de mémoire". Plus à l'écart, mais dans un rayon strictement inférieur à cent kilomètres, la seconde ceinture de cinq restaurants satellites à Lyon, Mâcon, Bourg-en-Bresse et, plus récemment, à Jassans-Riottier. "Tous ces établissements que je viens d'évoquer représentent des sources de revenus annexes. Ils ont été autant de greffes réussies sur ce qui constitue le noyau central de mes activités. Ils n'auraient pu exister sans cette locomotive en or, ce cour qui bat à Vonnas, ce restaurant étoilé qui reste ma raisin d'être" (Blanc, 2009, p. 94). Cette structuration spatiale révèle de la part de Georges Blanc une volonté de contrôle économique (en termes de recrutement, de formation et de management) du territoire. Ce contrôle renforce, d'une part, le sen- 
timent d'appartenance des différents membres du "système Blanc" et, d'autre part, l'effet de reconnaissance et d'appropriation de la clientèle, particulièrement locale et régionale (habitants permanents): "Les établissements sont à moins d'une heure de chez moi, cela me permet de tout contrôler. Nos maisons doivent avoir une identité forte, une âme, que seule la présence du chef peut conférer ${ }^{(5)}$." Ce qui n'interdit pas à G. Blanc, bien au contraire, dans une dynamique de visibilité et de reconnaissance internationale, d'exercer une activité lucrative de conseil culinaire et gastronomique : soutien technique et logistique à la production culinaire (fiches recettes) pour la compagnie aérienne Singapore Airlines, formation des personnels de cuisine pour le croisiériste Carnival Cruises ou conception de plats, durant cinq années, pour le restaurant Normandie de l'hôtel Oriental de Bangkok. Cette activité de conseil permet également à Georges Blanc de se constituer un réseau professionnel et commercial élargi.

\section{DU FAÇONNAGE D'UNE ATMOSPHÈRE GASTRONOMIQUE}

Cette concentration stratégique d'établissements et d'activités économiques est fondée sur une culture productive localisée. Elle est accompagnée d'une "culture de nourrir" dans laquelle s'expriment des habitudes culinaires et des préférences gustatives locales. Toutes deux valorisées par G. Blanc à travers la dif- fusion d'un discours et d'un imaginaire, elles permettent l'émergence d'une atmosphère gastronomique, qui va mettre en place les conditions nécessaires au développement local. Le façonnage de cette atmosphère gastronomique passe, d'une part, par une thématisation et, d'autre part, par une labellisation identitaire.

\section{L'édification du \\ "Village gourmand" ou le "Village dans le village" : la thématisation}

Au cœur du village de Vonnas, autour du restaurant 3 ț et de la place du Champ-de-Foire, Georges Blanc va progressivement constituer un "pôle" entièrement thématisé, dédié à la célébration du chef cuisinier et de sa cuisine. Comme l'écrit poétiquement le chanteur Henri Salvador dans le livre d'or du restaurant: "Ici, c'est comme le paradis, c'est tout 'BLANC'." Mû à la fois par la volonté d'unicité du lieu et par l'obsession de maîtriser l'environnement visuel et esthétique de ce lieu, il va créer une atmosphère favorable à la dégustation et aux plaisirs de la bonne chère, à la rencontre entre le chef cuisinier et les mangeurs, à la découverte d'un lieu gourmand original à la fois attractif par les habitants permanents et les habitants temporaires, les touristes.

Inspiré par le designer Raymond Loewy, concepteur de l'esthétique industrielle, G. Blanc ambitionne de faire rêver les mangeurs et les promeneurs dans le village: "Un sacré personnage ce Raymond Loewy et un homme délicieux. L'ami des plus grands de ce monde à son époque. J'adorais discuter avec lui. Il m'avait permis de comprendre toute l'importance de l'aspect environnemental pour notre maison de Vonnas : la présence de l'eau, de la verdure, des fleurs, de la lumière pour donner de l'éclat la nuit. Il me parlait d'espace, d'esthétique, et je l'écoutais toujours avec beaucoup d'intérêt, car j'étais vraiment en phase avec lui et avec ses idées. Le succès d'un établissement comme le nôtre tient dans une somme de détails déterminants. À commencer par l'aspect graphique de la cuisine dans l'assiette. Il faut que la présentation soit belle, qu'elle dégage des odeurs agréables, que ce soit très bon. Il faut ensuite que l'appréciation de la clientèle aille audelà du contenu de l'assiette, qu'elle porte sur le décor de la table, sur le cadre même du restaurant et sur les abords de l'établissement" (Blanc, 2008, p. 61). Aussi cherche-t-il à façonner une ambiance immatérielle, symbolique et diffuse, un "esprit des lieux" (ibid.) : "Le site, le lieu, son ambiance, son accueil, et surtout bien sûr la cuisine, tout cela a pour moi une égale importance. C'est en tout cas ce que j'ai ressenti très tôt : les gens reviennent dans un endroit qui a un passé, une histoire à raconter, une âme véritable, une réelle authenticité et des vraies valeurs dans l'assiette, et qui dispose - cerise sur le gâteau - d'un environnement particulièrement agréable. En ayant réussi, au fil des ans, à conjuguer tout cela, je pense qu'on a fait beaucoup pour asseoir la notoriété de la maison, sans toutefois perdre de vue 
que le succès n'est jamais acquis une bonne fois pour toutes, qu'il ne faut jamais s'endormir sur ses lauriers, mais au contraire s'efforcer de surprendre et d'étonner nos visiteurs" (id, p. 63).

G. Blanc va donc progressivement thématiser le village. Pour ce faire, il va acquérir, concentriquement, autour du restaurant 3 放, les biens immobiliers en vente pour les transformer en lieux gourmands en vue de former aujourd'hui un ensemble soigné et apprêté de plus de cinq hectares: "D'où mon idée de protéger le site en faisant les acquisitions nécessaires afin de constituer 'un village dans le village' voué à l'art de vivre, le tout dans un environnement harmonieux et préservé de 5,5 hectares. L'architecture, les couleurs, l'eau, la verdure, les fleurs, les lumières contribuent au charme $d u$ village $^{(6)}$."

Dès 1990, Georges Blanc rachète ainsi le café-épicerie-boulangerie de la famille Charvet-Guyennet et y réinstalle L'Ancienne Auberge. Progressivement, il va acheter dixsept maisons pour y aménager des hébergements, des espaces commerciaux et ludo-sportifs tout en cherchant à diversifier l'offre de restauration. "Depuis quarante ans, $j$ 'ai essayé d'agir, en permanence, sur l'aménagement de notre environnement, sans rien négliger, parce que j'ai toujours été persuadé qu'à qualité de cuisine sensiblement égale, le fait d'offrir à sa clientèle un décor de rêve, sortant de l'ordinaire, vous donnait un indéniable avantage. Un atout même décisif pour la fidéliser.
Dans la présentation du joyau, l'écrin compte autant que le joyau luimême" (Blanc, 2008, p. 62).

G. Blanc va porter une attention particulière à la clientèle touristique. Il développe l'hospitalité du "Village gourmand" afin de démultiplier son attractivité : "J'ai très vite compris que si vous êtes à la campagne, un hébergement de qualité, ajouté à la qualité de l'environnement, est la clé du succès $^{(7)}$." Ainsi, Georges Blanc comprend très vite la nécessité économique et financière de la complémentarité entre cuisine et hôtellerie : "Une fois aux commandes de l'établissement, $j$ 'ai voulu en augmenter la fréquentation. J'avais très tôt compris la complémentarité indispensable entre la restauration et l'hébergement. Il fallait offrir aux voyageurs qui faisaient étape ici, et qui venaient parfois de loin, à la fois le plaisir de la table et le confort la nuit. C'est ce que nous avons entrepris de proposer dans les années 1970. En prenant beaucoup de risques dans l'investissement, beaucoup plus que maintenant, je dois le dire, parce que notre volume d'activités était loin d'être celui d'aujourd'bui et que, du coup, les bases sur lesquelles nous bâtissions pour l'avenir étaient moins solides [...]. Nous partions d'une dizaine de chambres très simples, an confort limité, pour parvenir à cinq fois plus trente ans plus tard" (Blanc, 2008, p. 58).

Cette hospitalité passe par une accessibilité géographique de l'établissement. Georges Blanc sera, sinon le premier, l'un des premiers chefs cuisiniers à faire construire dès 1976 une hélistation, qui compte aujourd'hui environ 70 à 80 mouvements annuels. Mais c'est surtout l'accessibilité autoroutière qui va s'avérer être déterminante. Georges Blanc profitera en effet de son investissement politique à la mairie de Vonnas pour influer sur la réalisation d'une sortie d'autoroute desservant Vonnas et, dans le même temps, le "Village gourmand". "J'ai suivi un temps le même parcours que mon père, comme simple élu au conseil municipal de Vonnas, chargé du développement économique et de l'environnement. Un mandat passionnant durant lequel j'ai engagé, entre autres, des démarches pour que l'on obtienne une sortie d'autoroute sur l'A 40 Genève-Mâcon. À quoi pouvait servir une autoroute si on ne pouvait disposer d'accès et de sorties? J'ai consacré beaucoup de temps à faire du lobbying auprès des acteurs économiques et des élus de la région. Le combat fut long et rude. Mais c'est par un vote unanime, toutes tendances politiques confondues, que le conseil général, sous l'impulsion de Jacques Boyon et de Guy de La Verpillière, décida de l'utilité de cette bretelle et de sa réalisation. Avec des accents teintés d'un peu d'ironie (amis qu'importe!) certains, dans la région, la baptisèrent 'sortie Georges Blanc', en faisant bien sûr allusion à l'établissement qu'elle desservait. Je ne nie pas que nous en bénéficions, mais cet échangeur a profité et profite toujours largement à tout le monde, à l'ensemble des entreprises situées tout autour, comme aux touristes et aux parti- 
culiers dans leurs déplacements quotidiens" (id., pp. 83-86). Bien sûr, le lien particulier établi entre G. Blanc et son territoire a favorisé cette entreprise de lobbying: "Je pense que si je n'avais pas eu un enracinement profond, qui donne une certaine influence, cet échangeur n'aurait pas existé...” (interview G. Blanc).

\section{Bresse versus Dombes :}

\section{la labellisation identitaire}

Bien que localisé à la frontière géographique de la Bresse et de la Dombes, G. Blanc n'a de cesse d'affirmer, par son discours et son imaginaire, ses racines identitaires bressanes: "Vint l'adolescence... J'avais l'âge où l'on commence à mûrir, l'âge où l'on se pose un peu plus de questions sur l'avenir. Tout en aimant en savoir plus sur le passé. Celui de ma famille, par exemple, et sur toutes ces générations Blanc dont on avait remonté la trace jusqu'en 1510, ce qui faisait dix-huit an total. Un bel arbre généalogique qui prenait racine au hamean des Blancs à Marboz, en pleine Bresse, et qui s'épanouissait en bien d'autres branches qui ont permis à mes ancêtres d'essaimer dans toute la région” (Blanc, 2008, p. 31).

Dans la lignée de sa grand-mère et de sa mère, Georges Blanc privilégie les approvisionnements bressans, même si le chef cuisinier insiste très peu, à l'exception des volailles, sur l'origine géographique des produits agricoles alimentaires cuisinés : "Quand on a su se tisser un bon réseau de producteurs locaux nous avons la chance ici, en Bresse, d'être dans le voisinage du Val de Saône et de ses terres légères qui se prêtent bien à la culture marầchère-, nul n'est besoin de cultiver ou d'aller chercher soi-même ses herbes et ses plantes dans son propre jardin ou dans la nature" (id., p. 172).

L'implication de Georges Blanc dans la défense et la promotion de l'appellation d'origine contrôlée "volaille de Bresse" (obtenue le $1^{\text {er }}$ août 1957), largement affichée, est déterminante en termes d'identification culturelle. Le chef cuisinier intègre le Comité interprofessionnel de la volaille de Bresse (CIVB) en 1976 et en devient président en 1986 : "J'ai toujours été un ardent défenseur de la volaille de Bresse. Comme ma mère et ma grand-mère ont pu l'être avant moi, mais en des temps où notre célébrité locale n'avait pas trop besoin que l'on prenne sa défense, vu qu'elle n'était que très rarement attaquée" (id., p. 179). Dans son autobiographie, Georges Blanc qualifie lui-même son rôle au sein du CIVB de "médiateur" et d" "ambassadeur" (id., p. |8I) d'un produit agricole alimentaire haut de gamme et, consubstantiellement, d'une origine géographique et d'une identité. Il est dès lors possible de considérer que la relation Georges Blancvolaille de Bresse est intéressante et profitable dans les deux sens. Bien sûr, la vitalité économique et l'excellence culinaire et gastronomique que personnifie Georges Blanc rayonnent sur la volaille de Bresse qui, par effet d'osmose, se charge de ses qualités ; mais, finalement, l'image luxueuse et profondément enracinée de la volaille de Bresse, décrite dès 1825 par Brillat-Savarin comme "la reine des volailles et la volaille des rois" et qualifiée par le CIVB de "quatrième merveille gastronomique $d u$ monde" (après le foie gras, le homard et la truffe), participe à l'ancrage et à l'identification culturelle de G. Blanc et de son entreprise: "Très grosse notoriété de la volaille de Bresse, très grosse notoriété de Georges Blanc, on est très utile les uns aux autres" (interview CIVB).

G. Blanc aime particulièrement souligner deux éléments constitutifs de cette labellisation identitaire bressane. D'une part, le sentiment communautaire et la solidarité développés par l'ensemble de la filière - et lui-même - lors de l'épreuve de la crise aviaire: "Une fois encore, la Bresse se montra solide, solidaire, responsable. À aucun moment, les éleveurs ne cédèrent au découragement. Certes, il y eut une chute verticale des ventes, suite surtout à l'affolement assez compréhensible des consommateurs. Notre travail consista à les rassurer, donc à les informer, à la télévision, à la radio, dans les journaux, afin que leur confiance un moment ébranlée nous soit de nouveau acquise [...]. Dans son passé, lointain ou plus récent, la Bresse avicole a toujours su relever la crête avec courage et enthousiasme quant le sort lui fut défavorable et son destin incertain" (Blanc, 2008, pp. 185-186). D'autre 
part, la technicité et la qualité productive de la filière, qu'il encourage : “[...] J'ai tonjours eu le sonci, an cours de tous mes mandats successifs, de veiller à une certaine rentabilité pour que tous les acteurs de la filière vivent de cette volaille, depuis la reproduction du poussin jusqu'à l'abattage et la commercialisation" (id., p. 183). Il veille à nouer des relations privilégiées avec les producteurs : "C'est mon plaisir d'aider les agriculteurs, il y en avait un à l'auberge hier, un petit jeune" (interview G. Blanc). "On a des formations chaque année pour de nouveaux éleveurs et j'avais demandé à Georges s'il pouvait les accueillir en cuisine pour qu'ils voient comment leur produit allait être travaillé. Ça les motivait énormément !... Ils voient pourquoi le cahier des charges est aussi drastique. C'est des rencontres vraiment intéressantes... ils sont aussi très fiers d'être en contact avec un chef trois étoiles" (interview CIVB). Mais ce sont surtout les actions promotionnelles qui justifient l'élection de G. Blanc à la présidence du CIVB. En faisant la promotion de la volaille de Bresse, consciemment ou inconsciemment, volontairement ou involontairement, directement ou indirectement, Georges Blanc assure aussi sa promotion, celle de ses établissements et celle de son identité bressane. En France mais aussi, et surtout, à l'étranger.

Cette identité bressane, et cette identification à la volaille de Bresse, se traduit par le choix du volatile comme logo de l'entreprise que l'on retrouve sur la vaisselle, sur le linge de table et de toilette, sur le papier cadeau et d'emballage, sur les menus, les sacs... Cet "effet label" joue désormais à plein pour l'entreprise elle-même et, en particulier, sur l'image des produit vendus dans les commerces de Vonnas. En conséquence de quoi, les différentes boutiques de Georges Blanc (arts de la table, alimentation, vins et alcools, boulangerie-pâtisserie) ne désemplissent pas, qu'il s'agisse des produits signés et même des produits sélectionnés et conseillés par le palais aiguisé de Georges Blanc. La boutique gourmande propose des vins de Bourgogne issus des vignes de l'entreprise Blanc ou de producteurs locaux, les spécialités régionales comme les quenelles sont mises en valeur.

Ce label joue aussi pour les autres entreprises locales - du secteur de l'hôtellerie-restauration au premier chef, même bas de gamme - et pour le territoire. Il va lui permettre de se démarquer, de se différencier relativement par rapport aux autres territoires. L'excellence et le dynamisme associés à l'entreprise Blanc, par mimétisme, qualifient ainsi l'espace d'appartenance. "Quand le Tour de France est passé à Bourg, les élus m'ont dit : dis donc Georges n'accapare pas trop les journalistes avec tes volailles! parce que les journalistes, c'est ça qui les intéresse ici. Il y a la plasturgie à Oyonnax mais le public il n'a pas envie qu'on lui parle de la plasturgie" (interview G. Blanc et maire de Vonnas). Comment estil rendu accessible aux touristes ?

\section{LA CO-PRODUCTION D’UNE DESTINATION TOURISTIQUE GOURMANDE PAR LA THÉÂTRALISATION DE L'ATMOSPHÈRE GASTRONOMIQUE}

"Il y a trois esthéticiennes et six boulangeries à Vonnas! C'est bien plus qu'il n'en faut pour les habitants" (interview maire de Vonnas et G. Blanc). L'attrait touristique du village se développe et bénéficie indiscutablement à l'économie locale. Le parcours économique de G. Blanc est exemplaire dans sa capacité à transformer le village par le façonnage d'une atmosphère gastronomique qu'il va thêâtraliser afin de la rendre visible, lisible, préhensible, en fait "com-préhensible" par les touristes, les habitants permanents et les équipes du groupe Blanc. Celleci devient la vitrine du village mais aussi un espace d'intermédiation entre les touristes et le lieu, les touristes et le chef cuisinier (et son équipe), entre les touristes euxmêmes. Car les touristes ne se contentent pas de regarder le village, ils y habitent et y vivent une expérience gastronomique et touristique. $\mathrm{La}$ qualité de cette expérience est liée à son décor, à l'intrigue qui s'y raconte, aux relations qui s'y nouent (Filser, 2002). Ainsi G. Blanc théâtralise-til l'atmosphère gastronomique qu'il a réussi à faire émerger afin de favoriser les actions et les interactions avec les mangeurs qu'il ne manque jamais de saluer et avec qui il ne manque jamais de se faire photographier. 


\section{Georges Blanc :}

acteur central, metteur en scène emblématique

Dans l'histoire de la théâtralisation de l'atmosphère gastronomique, la scène qui se joue à l'occasion de l'obtention de la troisième étoile le 3 mars 1981 - et de son extrême médiatisation - va être déterminante. Cette troisième étoile est en effet une reconnaissance du monde professionnel et, plus encore, de la critique gastronomique. Elle reconnaît un niveau de qualité "supérieure" exceptionnel, un degré d'excellence rare : "J'étais le seul promu triplement étoilé de ce millésime. Les retombées furent immédiates et même durables. Je ne pouvais deviner à cette époque qu'il n'y aurait pas d'autres trois étoiles dans les deux années qui suivront... En cette même année, le guide Gault et Millau me désignait 'meilleur cuisinier de l'année'. Quatre ans plus tard et pour la première fois dans les annales de son classement, il m'attribuait la note de 19,5 ! [...] Dans son journal de 20 beures, Patrick Poivre d'Arvor annonça cette promotion. Ce fut, je crois, la première fois qu'un présentateur $d u$ journal télévisé donnait ce genre d'information, surtout à une heure de grande écoute. [...] J'eus le sentiment de passer, auprès du grand public, de l'anonymat presque total à une forme de vedettariat que je n'aurais jamais cru concevable vingt-quatre heures plus tôt. [...] Tout ce battage déclencha un appétit médiatique incroyable. Je reçus très vite moult propositions en tous genres qui allaient de l'ouverture de restaurants de par le monde à des partenariats comme consultant pour des groupes internationaux" (Blanc, 2008, pp. 7475).

G. Blanc est devenu un acteur central. La directrice de l'office de tourisme de Chalaronne Centre confie en souriant: "Quand les gens téléphonent et demandent 'où est Châtillon ?', on répond 'à côté de Vonnas' alors que cette commune est plus grande" (interview OT de Chalaronne Centre).

Il est cependant à souligner que l'investissement d'un acteur privé, aussi emblématique soit-il, dans le développement local n'est pas du goût de tous : "Ça amène des critiques, des jalousies. Quand j'ai fait l'enfouissement des réseaux aériens... il fallait dépoussiérer le paysage. On $m$ 'a dit: 'Mais non Georges, ça va coûter très cher. On n'aura pas de subventions, on n'est pas une commune classée'... J'ai pu convaincre ceux qui s'occupent des subventions qu'on n'est peut être pas classés mais qu'on est une commune touristique. Comme pour la signalisation. Et on a pu faire cet alignement de candélabres en bronze... Eh bien les gens, ils ont critiqué, ils disaient: 'Georges Blanc il fait cela pour son commerce...' alors que toutes les communes investissent dans la qualité environnementale maintenant" (interview G. Blanc). Le maire actuel entend cependant bien valoriser cette manne, de même que les acteurs du tourisme. Des rapprochements sont en cours avec l'office de tourisme de la communauté de communes de Chalaronne Centre : une nouvelle structure est implantée à Vonnas, le Pavillon du tourisme, avec un poste à mi-temps financé par la commune de Vonnas. G. Blanc participe à cette nouvelle structure et s'y investit : "Ses services commerciaux ont rencontré ceux de l'office, ils ont visité son établissement... on sent de sa part une volonté de travailler avec les professionnels du tourisme" (interview OT de Chalaronne Centre). Cet investissement est guidé par le désir de favoriser l'attractivité touristique du village considéré par G. Blanc comme un "patrimoine" : "On n'a pas de patrimoine architectural mais on a un patrimoine touristique conséquent" (interview G. Blanc).

\section{L'entrée en scène \\ et le jeu interactif \\ des acteurs touristes}

La théâtralisation de l'atmosphère gastronomique participe directement $\mathrm{du}$ rayonnement touristique de Vonnas. Ainsi, pour de nombreux touristes, le restaurant $3 \hbar$ et le Village gourmand constituent le but du voyage: "J’apprécie quand les clients me disent: 'Nous sommes venus vous voir et découvrir Vonnas avec une attente formidable. Eh bien, franchement, nous n'avons pas été déçus.' C'est le plus beau compliment que l'on puisse me faire" (Blanc, 2008, p. 63). Vonnas accueille aujourd'hui environ 120000 touristes par an. Si aucune statistique n'est disponible pour confirmer l'impact clé de l'entreprise Blanc sur le développement touristique de Vonnas, on peut toutefois affirmer que l'attractivité tou- 
ristique de Vonnas est très liée à l'univers gastronomique Blanc. Le Pavillon du tourisme propose même désormais un circuit de découverte du patrimoine local, dont la première étape sont les établissements Blanc. En ce sens, le restaurant 3 灾 et, plus largement, le Village gourmand peuvent être considérés comme destination touristique gourmande.

Le passage d'un lieu à une destination touristique a été analysé par quelques chercheurs qui mettent en évidence plusieurs phénomènes participant à ce processus.

Le premier phénomène à l'œuvre concerne les projections des visiteurs. Le lieu devenu destination est représenté et projeté par les touristes. La destination touristique est "un territoire qui se prête à une interprétation touristique", selon la définition de Jean-Marie Hazebroucq (2009). Dans le même esprit, Philippe Violier définit la destination touristique comme "une représentation d'un lieu ou d'un espace dans lequel le touriste se projette pour mettre en ceuvre un projet de recréation (repos, jeu, découverte) et comme espace vécu, c'est-à-dire perçu et pratiqué par le touriste, la destination constitue le lien entre le monde et le lieu" (Violier, 2009). Il existe donc un phénomène de projection, de curiosité, d'imagination et d'interprétation qui participe à la transformation du lieu en une destination.

Le deuxième phénomène à l'œuvre concerne les liens sociaux qui s'y nouent. La destination touristique est un terrain de rencontre entre, d'une part, habitants permanents et habitants temporaires, et d'autre part entre touristes. C'est un lieu de cohabitation. La destination touristique est ainsi une scène de confrontation du local et du global, du microcosme et du macrocosme, à l'articulation entre ouverture et fermeture, co-construit par la tension entre grandes tendances générales et menues contre-tendances localisées.

La destination touristique est par ailleurs un lieu impulsé, valorisé et promu, du point de vue touristique, par un acteur central qui peut être privé ou public, individuel ou collectif. Philippe Violier et Antonio Manuel Zarate (2007) insistent sur le rôle de cet acteur central capable d'animer un jeu d'acteurs locaux, et de valoriser des qualités du lieu peu perçues comme telles. En effet, les deux auteurs rejettent l'idée d'un "existant antérieur qui justifie d'emblée une politique touristique" et d'un tourisme qui serait "seulement la mise en tourisme d'un stock non exploité" (Violier et Zarate, 2007. p. 148) et invitent à analyser la mise en tourisme "en termes d'interrelations entre des jeux d'acteurs et des qualités des lieux dans un contexte local et global précis” (id., p. 149). La destination touristique est coconstruite au croisement des projets et des stratégies du système d'acteurs touristiques et des pratiques des touristes.

Le lieu touristique est un lieu ordinaire et du quotidien qui subit une transformation touristique qui le fait passer dans l'extraordinaire et le hors-quotidien, pour reprendre les expressions formalisées par Mathis
Stock et Philippe Duhamel (2005). La destination touristique est un lieu transformé dans le temps des mobilités temporaires et dans l'espace des aménagements touristiques. Son développement est lié à la capacité des acteurs à comprendre le passage à l'extraordinaire et au hors-quotidien dans leur structuration, la mầtrise des différentes phases de développement de la destination, dans la gestion de la dynamique et de l'itinéraire de la destination et dans l'adaptation continue aux évolutions des pratiques et des représentations sociales des touristes. À Vonnas, ce passage est favorisé par la théâtralisation opérée par Georges Blanc.

Ces travaux contribuent donc à caractériser non seulement le processus de transformation d'un lieu (gourmand) en destination touristique (gourmande) mais aussi à caractériser ce qu'est cette dernière. Ils nous permettent d'affirmer qu'aujourd'hui Vonnas est bien une destination touristique gourmande. Le village est en effet un lieu gourmand qui a été impulsé touristiquement par G. Blanc. L'obstination de la signalisation routière est significative : la commune de Vonnas n'était pas indiquée sur le tronçon autoroutier de l'A 404, c'est le chef cuisinier qui a pris le dossier en main pour l'obtenir. Vonnas est incontestablement un lieu projeté par les touristes. Il suffit pour s'en convaincre de lire les avis des visiteurs: "Nous étions très impatient de venir à Vonnas découvrir la cuisine de Georges Blanc"; "J'ai fêté mes 40 ans à Vonnas, une surprise organisée par 
ma chère et tendre, elle ne pouvait pas choisir mieux... Un grand nom pour une grande occasion, quoi de mieux..." ; "Ne pas pénétrer une fois dans sa vie dans l'antre de la gastronomie française eût été regrettable et je n'ai rien regretté( $e^{(8)} . .$. " Il y a une part de rêve dans le fait de réserver chez Blanc, que ce soit dans le restaurant étoilé ou à L'Ancienne Auberge. Et la carte signée remise en main propre par le chef est ramenée comme un souvenir mais aussi un témoignage du fait que l'on est gastronome, que l'on connaît le chef, que l'on a été transformé par cette dégustation et cette visite du lieu gourmand. '[...] J'aime être présent pour les hôtes. D'ailleurs, après chaque repas, je vais à leur rencontre. C'est un moment que j'apprécie. Un restaurant même triplement étoilé doit être un lieu de convivialité(9)." Par le truchement du chef cuisinier, le lieu permet de rencontrer et de faire se rencontrer des gastronomes de tous pays. Le monde entier connait Georges Blanc et les touristes affluent de tous les continents. Il est intéressant à cet égard de s'arrêter sur les données de fréquentation fournies par le Pavillon du tourisme de Vonnas. Ces données sont très parcellaires puisque à l'époque de notre enquête celui-ci commençait juste à se restructurer et entamait la collecte d'informations. Pour autant, nous pouvons déjà mentionner que, sur les deux mois précédant notre observation participante (mai et juin 2012), plus de $16 \%$ des visiteurs qui poussent la porte pour demander des informations pratiques sur
Vonnas et sa région, les fêtes et manifestations (et non sur le restaurant) sont d'origine étrangère : Hong Kong, Australie, Corée, Allemagne, Royaume Uni, Suisse, Japon, ÉtatsUnis, Canada ou Belgique sont mentionnés comme origines géographiques des touristes. La mise en tourisme d'un lieu passe enfin par l'aptitude des acteurs de la destination à faire vivre une expérience aux visiteurs (Pine et Gilmore, 1998), expérience singulière qui les éloigne de leur ordinaire. La théâtralisation de l'atmosphère gastronomique est ici affaire de production d'expérience (Filser, 2002). Dans le cas de Vonnas, cette théâtralisation repose d'abord sur la volonté continue d'étonner et de surprendre. Georges Blanc met en scène la cuisine et propose aux touristes de vivre une expérience unique à Vonnas: "La magie doit opérer aussi bien dans la salle que dans l'assiette ${ }^{(10)}$." C'est ainsi que les mangeurs sont conduits depuis la réception vers les salles à manger tout en passant devant les cuisines largement vitrées, de manière à bien mettre en évidence la permanence et la vitalité de l'histoire familiale (cuisines dirigées par Frédéric Blanc, le fils aîné) et culinaire de la maison.

De plus, des offres packagées sont proposées pour le week-end avec cours de cuisine et initiation à la dégustation avec Fabrice Sommier, "meilleur ouvrier de France", qui permettent aux touristes de "mettre la main à la pâte" : "Je suis persuadé que, face à qualité de cuisine sensiblement égale, les clients ont toujours envie de revenir dans un endroit où ils se sentent bien, respectés et choyés. 'Choyés', ce mot correspond parfaitement à ce que pratiquaient les mères Blanc et je mets toujours un point d'honneur à respecter cette philosophie ${ }^{(11)}$."

Cette expérience va au-delà du culinaire et du gustatif: "Les gens viennent à Vonnas pour y viure une expérience unique. Ils peuvent rester 2 ou 3 jours, se rendre au spa et/ou au golf qui se trouve à proximité, dormir dans l'une de nos 60 chambres, dîner au restaurant et déjeuner dans l'ancienne auberge, voire dans l'un des petits restaurants que j'ai développés dans les villes avoisinantes $^{(12)}$." Elle perdure en dehors des lieux de restauration et d'hôtellerie, dans les rues et sur la place du Champ-de-Foire où il est alors possible d'observer et de participer à un étonnant ballet où se croisent et se rencontrent Georges Blanc, ses cuisiniers, son fils aîné Frédéric au premier chef, qui entrent et sortent des différents établissements et traversent les espaces publics extérieurs avec sourire et décontraction tout en arborant fièrement leurs vêtements professionnels, les habitants permanents (notamment le dimanche, lors de la promenade dominicale) et les touristes. Cette théâtralisation est renforcée par des scènes interactives plus ou moins improvisés durant lesquelles G. Blanc se laisse photographier avec les touristes devant le restaurant 3 放 ou L'Ancienne Auberge et se prête à des signatures de menus, de cartes et de livres qu'il a écrits, où il raconte 
son histoire, sa famille, sa passion, sa cuisine, entretenant avec chaque lecteur un lien singulier que seule la littérature est en mesure de proposer.

Les travaux de Holbrook et Hirschman (1982) montrent que la production d'une expérience de consommation est une mise en scène et tient au décor (la théâtralisation), à l'intrigue (le récit) et à l'action (les relations qu'entretient le consommateur avec le produit) (Filser, 2002). L'unité d'action est donc nécessaire. Nous pourrions ajouter l'unité de lieu pour rejoindre à la fois l'histoire de l'édification du Village gourmand et les recommandations du théâtre classique. Manquerait bien sûr l'unité de temps, mais quiconque se promène et habite à Vonnas reconnaît qu'ici le temps s'est arrêté et que L'Ancienne Auberge projette le touriste au début du siècle dans le temps des mères Blanc.

Dans le but de favoriser la co-production de l'expérience, la théâtralisation de G. Blanc vise à attiser la curiosité du touriste en lui proposant d'assouvir son désir de découverte (d'un autre et d'un ailleurs, ici par la dimension gustative) qui constitue l'un des motifs justifiant le déplacement touristique. Pour ce faire, selon une logique balistique, G. Blanc procède à la création d'aménagements (une allée Jean-Louis-Blanc, une placette Élisa-Blanc, un itinérairemémoire paysager de promenade...) et d'expositions (photographies familiales, menus historiques, plaques commémoratives...) qui informent, avec des effets narratifs et visuels, le touriste, suscitent l'esquisse d'une homologie (Cochoy, 20I I, p. 45) et finalement invitent le touriste à se positionner et à s'inscrire dans le temps et dans l'espace de cette théâtralisation. La curiosité est à la fois construite et activée (Cochoy, 20 I I, p. 55) par G. Blanc. Pour ce faire, il manipule une matière discursive (littéraire) et imaginaire (cinématographique-théâtrale) à la fois réelle et idéelle, à la fois matérialisée et symbolique, à la fois concentrée et diffuse. Le touriste acquiert ainsi une série de compétences qui lui permettront de jouir au mieux de son expérience en s'y investissant, en l'in-corporant, en la comprenant et, finalement, en la co-produisant. Cette acquisition de compétences facilite alors la rencontre, l'échange et, plus encore, l'interaction entre les touristes et le chef cuisinier (et son équipe), entre les touristes et les habitants permanents et entre les touristes euxmêmes. Elle produit une socialisation fondée sur le partage d'un même engouement. La découverte théâtralisée par G. Blanc est à la fois celle d'une construction intellectuelle et celle d'un "trésor".

Nous connaissons bien les travaux de Marshall (1890) et surtout leur potentiel à conceptualiser des dynamiques de croissance endogène des territoires assises sur la vitalité d'un réseau territorialisé d'entreprises, sur celle d'un marché du travail local et sur la créativité des acteurs de ce même territoire. Ces dynamiques de croissance sont celles qui sont en jeu dans les districts industriels de l'Italie du Nord (Becattini, 1992), dans les systèmes productifs localisés (Courlet,
2002) et aussi dans les clusters (Porter, 1998). Les concepts théoriques de Marshall trouvent un large écho auprès des chercheurs qui s'interrogent sur les avantages de la proximité géographique et organisationnelle (Torre, 20I0) mais aussi ceux de la proximité culturelle, d'une histoire commune, de liens sociaux (Pecqueur, 2000) et ceux d'un capital social partagé sur un territoire (Nahapied, Goshal, 1998). Grâce aux nombreuses interactions qu'ils établissent, les acteurs d'un territoire développent des capacités d'apprentissage importantes qui se transforment en une compétence centrale pour le territoire dont Corinne Van der Yeught (2008) montre bien l'importance pour le management des destinations touristiques. La proximité géographique facilite ces interactions non seulement parce qu'elle diminue les coûts et les temps de transport mais aussi parce qu'elle les inscrit dans une dynamique réticulaire locale. La prédominance d'une activité permet en outre de créer un marché du travail et aussi d'animer un réseau de sous-traitants : les entreprises profitent de la disponibilité de compétences professionnelles sur place. L'histoire locale, la culture, les interactions sociales constituent les bases d'un capital social qui favorise une dynamique de création de connaissance. Les mécanismes à l'œuvre ont été largement étudiés dans l'industrie manufacturière mais aussi dans le tourisme (Fabry, 2009). Pour autant, nous disposons de peu d'éléments sur les mécanismes à l'œuvre dans ce qui constitue pourtant l'essence même 
du tourisme : l'association des touristes à cette dynamique. Les recherches sur les "clusters tourisme" (Jackson et Murphy, 2006 ; Jackson, 2006 ; Kim et Wicks, 20 I0) ou la compétitivité des destinations touristiques par la mise en réseau des acteurs et l'ancrage sur les ressources locales (Ritchie et Crouch, 2003 ; Nordin, 2003 ; Cunha et Cunha, 2005 ; Alvarez Gonzalez et Gonzalez Morales, 2006) restent, comme l'ont montré Clergeau et Violier (20। I), très orientées sur l'offre. L'économie touristique présentée dans ces travaux est une économie de l'offre. Or, le tourisme est bien une économie de coproduction. En ce sens, sa compréhension mérite que le touriste soit replacé au centre de la dynamique $\mathrm{du}$ territoire touristique et que les mécanismes qui soutiennent la relation co-productive entre les touristes et le territoire touristique soient explicités. C'est en ce sens que nous comprenons la spécificité d'une atmosphère gastronomique théâtralisée qui associe les touristes gourmands à la dynamique de compétences du lieu gourmand. Et c'est ce qui nous amène à définir l'atmosphère gastronomique comme l'accumulation de savoirs et de connaissances gastronomiques et liés au goût qui favorise l'apprentissage et l'acquisition de compétences par les protagonistes de la co-production d'une expérience touristique. L'atmosphère “industrielle" gastronomique à laquelle la famille Blanc a contribué est théâtralisée, rendue accessible et finalement partagée avec les touristes.

Nous appuyant sur les définitions que donne Hamel (1994) de la compétence stratégique, nous considérons ainsi que le territoire de Vonnas voit la concentration, la diversification et le développement d'apprentissages constitués de connaissances tacites et explicites enracinées dans la culture locale et qui imprègnent l'atmosphère locale, auxquels une savante théâtralisation permet d'associer les touristes. La théâtralisation sert ici de pont entre les mondes : celui des habitants permanents de Vonnas et celui des touristes. L'atmosphère gastronomique est sous-tendue par des discours, des imaginaires, des valeurs, des pratiques et des représentations sociales, mais aussi des savoirs et des compétences, que la théâtralisation rend accessibles aux touristes du monde entier qui trouvent dans ce Village gourmand de quoi nourrir une expérience unique. Si cette atmosphère est mise en scène par Blanc, nous devons souligner qu'elle est enrichie par les touristes. Ceux-ci, par leurs pratiques, ou leurs retours d'expérience, participent à cette dynamique. C'est en ce sens que nous parlons ici d'une atmosphère gastronomique, considérant que les acteurs du territoire de Vonnas, et G. Blanc tout particulièrement, en tant qu'acteur central, valorisent une culture productive et une "culture de nourrir" avec et pour les touristes.

\section{CONCLUSION}

Le cas de Georges Blanc à Vonnas nous a conduits à proposer et à caractériser l'atmosphère gastronomique d'un lieu gourmand comme élément fondamental de sa transformation en une destination touristique gourmande. Sa savante théâtralisation autorise une visibilité tant interne qu'externe (un rayonnement), une lisibilité optimale, corrélativement une meilleure appropriation réelle et symbolique et, finalement, une réelle co-production. Cette approche nous conduit donc à identifier l'économie touristique comme une économie de la co-production et permet d'envisager des pistes de travail sur la co-production touristique comme facteur de compétitivité des destinations.

Notre méthodologie d'étude de cas, ancrée dans l'observation participante et dans les données du terrain et interrogeant les théories existantes, favorise l'innovation théorique (Guillemette, 2006). Mais elle constitue également la limite de notre recherche actuelle. En effet, les dynamiques économiques des territoires sont plurielles et une généralisation serait ici prématurée. Pour autant, notre recherche offre des pistes de réflexion à la fois pour les praticiens et pour les chercheurs sur les modalités de transformation d'une atmosphère économique locale en une atmosphère touristique locale.

Celle-ci pourrait s'apparenter à une forme de "disneylandisation" si elle ne reposait sur une tradition familiale ancrée et reproduite, une culture productive et une identité locales. Elle pourrait être assimilée à une forme de marketing de l'authentique et de culture de la nostalgie, pour reprendre les termes de Cova 
et Cova (200I), si elle ne s'appuyait sur des savoirs locaux, des pratiques et des compétences locales, une atmosphère "industrielle" orientée vers la gastronomie. Et c'est là tout l'enjeu, toute l'originalité et toute la singularité de la construction de cette atmosphère gastronomique.

\section{NOTES \\ (I)}

http://unw.georgesblanc.com/maj/images/pdf/ museographie.pdf [consulté en septembre 2012].

(2) Revue du Touring Club de France, avril 1930, n²5, pp. II2-1। 3.

(3) "Georges Blanc est-il exceptionnel ?", Entreprendre, octobre 20I I, pp. 48-49.

(4) L'“assaisonnement liquide des mets", selon son expression

(5) Entreprendre, op. cit. (note 3), 2011.

(6) Le Chef, n 21 I ("Georges Blanc, 30 ans de trois étoiles"), mars 2010.

(7) "Portrait de chef: Georges Blanc", Culinaire Saisonnier, printemps 2010, p. 4.

(4) Témoignages Tripadvisor, février 2013.

(9) Entreprendre, op. cit. (note 3), 201 I.

(10) Entreprendre, op. cit. (note 3), $201 \mathrm{l}$.

(I I) Le Chef, op. cit. (note 6), 2010.

(12) Entreprendre, op. cit. (note 3), 201 I.

\section{RÉFÉRENCES BIBLIOGRAPHIQUES}

\section{José Antonio Alvarez Gonzalez et Dra}

Olga Gonzalez Morales, "'L'apprentissage, l'innovation et la compétitivité dans les clusters touristiques : une étude comparée entre l'Espagne et l'Italie", Revue d'économie régionale et urbaine, $n^{\circ}$ 4, 2006.

Jean-Luc ARrÈGLE, Rodolphe DURAND et Philippe VERY, "Origines du capital social et avantages concurrentiels des firmes familiales", M@n@gement, vol. 7, n², 2004.

Giacomo BeCATtINI, "Le district marshallien : une notion socioéconomique", dans Georges BENKO et Alain LIPIETZ, Les Régions qui gagnent, Puf, 1992

Georges BLANC, La Vie en Blanc, éditions GSL, 2008.

Mark C. BOLINO, William H. TURNLEY et James M. BLOODGOOD, "Citizenship behavior and the creation of social capital in organizations", The Academy of Management Review, vol. 27, $n^{\circ}$ 4, 2002.

Pierre BOURDIEU, "Le capital social. Notes provisoires", Actes de la recherche en sciences sociales, vol. 3I, 1980.

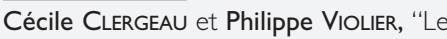
concept de cluster est-il soluble dans le tourisme ? Une approche conceptuelle", communication à la $I^{\text {re }}$ Conférence intercontinentale en intelligence territoriale, IT

Gatineau, Québec, 201।.

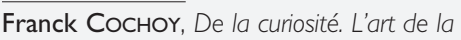
séduction marchande, collection "Individu et Société", Armand Colin, 201 I.

Claude COURLET, "Les systèmes productifs localisés. Un bilan de la littérature", Études et recherches sur les systèmes agraires et le développement, vol. 33, 2002.

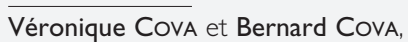

Alternatives marketing. Réponses marketing aux nouveaux consommateurs, Dunod, 2001

Sieglinde Kindl DA CUNHA et João Carlos DA CUNHA, "Tourism cluster competitiveness and sustainability: proposal for a systemic model to measure the impact of tourism on local development", Brazilian Administration Review, vol. 2, $n^{\circ}$ 2, juillet-

décembre 2005.

Albert DAVID, Armand Hatchuel

et Romain LaUfer (dir.), Les Nouvelles

Fondations des sciences de gestion, Vuibert, 2000

Fabien ÉLOIRE, Les réseaux interorganisationnels dans la restauration lilloise. Une approche néo-structurale du marché et des processus sociaux, thèse de doctorat en sociologie, Université de Lille I, 2009.

Kathleen M. EISENHARDT, "Building theories from case study research", The Academy of Management Review, vol. 14, n 4, 1989.

Nathalie FABRY, "Clusters de tourisme, compétitivité des acteurs et attractivité des territoires", Revue internationale d'intelligence économique, n I, 2009.

Marc FILSER, "Le marketing de la production d'expérience : statut théorique et implications managériales", Décisions marketing, $n^{\circ} 28,2002$.

François GUILLEMETTE, "L'approche de la Grounded Theory; pour innover ?",

Recherches qualitatives, vol. 26, n I, 2006.

Mark GranovetTER, "Economic action and social structure: the problem of embeddedness", American Journal of Sociology, vol. 91, n³, 1985. 


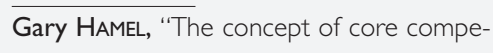
tence", dans Gary Hamel et Aime HeENE

(dir.), Competence-Based Competition, John

Wiley and Sons inc., 1994.

Jean-Marie HaZebroucQ, "De l'espace touristique à la 'destination touristique' : un territoire qui se projette", dans Jean-Pierre LEMASSON et Philippe VIOLIER (dir.),

Destinations et territoires 1. Coprésence à

l'œurre, Presses de l'université du Québec /

Téoros, 2009.

Morris B. HOLBROOK et

Elizabeth C. HIRSCHMAN, "The experiential aspects of consumption: consumer fantasies, feelings and fun", Joumal of Consumer Research, vol. 22, 1982.

Julie JACKSON, "Developing regional tourism in China: the potential for activating business clusters in a socialist market economy", Tourism Management, vol. 27, n 4, 2006.

Julie JACKSON et Peter MurPHY, "Clusters in regional tourism: an Australian case", Annals of Tourism Research, vol. 33, $n^{\circ} 4$, 2006.

\section{Namhyun KIM et Bruce E. WICKS, \\ "Rethinking tourism cluster development models for global competitiveness", \\ International Council on Hotel Restaurant and Institutional Education Conference, 2010 [en \\ ligne]. À partir de \\ [http://scholarworks.umass.edu/refereed/C HRIE_20 I0/Friday/28].}

Alfred Marshall, Principes d'économie politique, traduction de Principles of Economics (1890), Gordon \& Breach, 1971. Janine NAHAPIET et Sumantra GHOSHAL, "Social capital, intellectual capital and the organizational advantage", The Academy of Management Review, vol. 23, n², 1998.

Sara NoRDIN, Tourism Clustering and Innovation. Paths to Economic Growth and Development, European Tourism Research Institute, Östersund, Sweden, 2003.

Bernard PeCQUeUr, Le Développement local, Syros, 2000.

B. Joseph PINE et James H. GILMORE,

"Welcome to the experience economy", Harvard Business Review, juillet-août 1998.

Karl POLANYI, La Grande Transformation. Aux origines politiques et économiques de notre temps, Gallimard, 1983. (Traduction de The Great Transformation, publié à New York en 1944.)

Michael E. PORTER, "Clusters and the new economics of competition", Harvard

Business Review, novembre 1998.

Fabien REIX, "L'ancrage territorial des créateurs d'entreprises aquitains : entre encastrement relationnel et attachement symbolique", Géographie, économie, société, vol. 10 , $n^{\circ}$ I, 2008.

John Raymond Brent RitCHIE et Geoffrey Ian $\mathrm{CrOUCH}$, The Competitive Destination: a Sustainable Tourism Perspective, Cabi

Publishing, 2003.

Mathis Stock et Philippe DuHAmel, "A practice-based approach to the conceptualisation of geographical mobility", Belgeo (revue belge de géographie), n I-2, 2005.

André TORRE, "Jalons pour une analyse dynamique des proximités", Revue d'économie régionale et urbaine, $n^{\circ} 3,2010$.

Philippe VIOLIER, "Proposition pour un modèle d'analyse de la dynamique des lieux touristiques", dans Jean-Pierre
LEMASSON et Philippe VIOLIER (dir.), Destinations et territoires 1. Coprésence à l'œuvre, Presses de l'université du Québec / Téoros, 2009.

\section{Philippe VIOLIER et Antonio Manuel}

ZARATE, "Politiques urbaines du tourisme", dans Philippe DuHAmel et Rémy KNafou (dir.), Mondes urbains du tourisme, Belin, 2007.

Corinne VAN DER YeUGHT, "Les apports du développement durable au management stratégique des destinations touristiques", Conférence annuelle de l'Association internationale de management stratégique (AIMS),

Nice, 2008.

Robert K. YIN, Case Study Research: Design and Methods, Sage Inc., 2003 